\title{
ESTUDO COMPARATIVO DA AÇÃO INIBITÓRIA DA MANGIFERINA E DA ANILINA NA CORROSÃO DE AÇO CARBONO 1010 EM MEIO ÁCIDO
}

\author{
JORGE EDSON PINHEIRO DOS SANTOS, \\ Marcelo Valente Monteiro Parente, Caroline de Goes Sampaio \\ Instituto Federal de Educação, Ciência e Tecnologia do Ceará (IFCE) \\ Campus de Maracanaú \\ $<$ jepsa-015@hotmail.com> \\ <marceloparente2@gmail.com>,<carol-quimica@hotmail.com>
}

DOI: $10.21439 /$ conexoes.v10i5.1162

\begin{abstract}
Resumo. Controlar, ou inibir cineticamente o processo corrosivo tem se tornado um grande desafio para a ciência. Devido a este fato, tem-se buscado estudar os diversos tipos de interações entre material e meio, pois, se sabe que o processo corrosivo depende não só do próprio material, mas também das condições em que o mesmo fica exposto. Sabe-se que a utilização de inibidores constitui um dos métodos alternativos de proteção contra a corrosão. No entanto, há uma preocupação crescente sobre a toxicidade da maior parte dos inibidores de corrosão. Recentemente, vários trabalhos têm sido realizados que comprovam a ação inibidora de extratos de plantas frente à corrosão de metais. Desta forma, este estudo tem por objetivo realizar um comparativo entre o efeito da mangiferina e da anilina na ação inibitória da corrosão em meio ácido. Neste trabalho, foram realizados ensaios de perda de massa coadjuvados com ensaios eletroquímicas em meio ácido. Os resultados obtidos mostraram que o aço carbono exposto neste meio adicionado de mangiferina e anilina apresentou uma redução no processo de perda de massa, e uma redução dos potencias tanto anódicos como catódicos, confirmando que a mangiferina apresenta uma ação do tipo misto sobre o substrato metálico, já que a anilina com resultados semelhantes apresenta esta ação.
\end{abstract}

Palavras-chaves: Corrosão, Inibidor, Mangiferina.

\begin{abstract}
To control or to kinetically inhibit the corrosive process has become a major challenge for science. In fact, most of researchs have been made on interactions between the material and the environment, because it is known that the corrosion process depends not only on the material itself, but also on the conditions under which it is exposed. The use of inhibitors is one of the alternative methods of corrosion protection. However, there is growing concern about the toxicity of most corrosion inhibitors. Recently, several studies have been conducted that demonstrate the inhibitory action of plant's extracts on the metal's corrosion. Thus, this study aims to carry out a comparison between the effect of mangiferin and aniline in the inhibitory action of corrosion in an acid medium. In this study, weight loss tests were made by electrochemical technique's assitence in an acid medium. The results showed that the exposed carbon's steel in this medium, added mangiferin and aniline, got reduction on the weight loss process. Additionally, minors potential's values were observed for both anode and cathode, confirming that mangiferin presents an action of mixed type on the metallic substrate, since aniline with similar results showed the same action.
\end{abstract}

Keywords: RCorrosion, Inhibitor, Mangiferin. 


\section{INTRODUÇÃO}

Controlar, ou inibir cineticamente o processo corrosivo tem se tornado um grande desafio para a ciência ao longo dos anos. Devido a este fato, tem-se buscado estudar os diversos tipos de interações entre material e meio, pois, é sabido que o processo corrosivo depende não só do próprio material, mas também das condições em que o mesmo fica exposto (GENTIL, 2013).

Uma das formas de combater o efeito corrosivo é a utilização de inibidores que é um dos melhores métodos de proteção contra a corrosão em metais (EDDY; ODOEMELAM, 2008). No entanto, há uma preocupação crescente sobre a toxicidade desses inibidores. $\mathrm{O}$ efeito tóxico, não afeta somente os organismos vivos, mas também contamina o ambiente que, consequentemente, pode gerar problemas irreversíveis ao ecossistema afetado (EDDY; ODOEMELAM, 2008; EL-ASHRY et al. 2006).

Com isso, nota-se a necessidade do uso de inibidores de corrosão naturais, que não agridam o meio ambiente. Recentemente, vários estudos têm sido realizados sobre a inibição da corrosão de metais a partir de produtos obtidos de extrato de planta (SETHURAN; RAJA. 2006).

A Mangiferina (2-C-b-D-glicopiranosil-1,3,6,7tetrahidroxi-xantona) é um composto polifenólico amplamente encontrado em pteridófitas e angiospemas, principalmente nas famílias Gentianaceae e Iridaceae, presentes predominantemente nas folhas e na casca do caule. Em mangueiras, a mangiferina é um dos constituintes fenólicos majoritários, podendo ser detectada nas folhas, casca do caule, fruto e raízes.

A Mangiferina apresenta um grande potencial antioxidante, pois apresenta uma capacidade elevada de absorção de radicais oxigênio, devido ao fato de apresentar zonas de radicais hidroxila. Relatos apresentados na literatura (CANUTO, 2009) mostram que a capacidade antioxidante da mangiferina é mais potente que a do ácido ascórbico e da vitamina E.

Como forma de comparativo entre compostos de ação inibitória semelhante, foi utilizada a anilina que é um composto orgânico que apresenta ação inibitória do tipo misto e que tem como mecanismo de interação com o substrato metálico um método de adsorção como já relatado na literatura (VRACAR; DRAZIC, 2002).

Portanto, este trabalho tem como objetivo realizar um comparativo entre o efeito da mangiferina e da anilina na ação inibitória da corrosão em meio ácido.

\section{MATERIAIS E MÉTODOS}

A substância Mangiferina utilizada nesse trabalho foi cedida pelo Laboratório de Química Orgânica da Universidade Federal do Ceará - UFC. A mesma foi obtida a partir de extração do caule da planta Mangifera indica L. (mangueira), utilizando o etanol como solvente extrator. O procedimento de obtenção e purificação da Mangiferina está devidamente apresentado na literatura (SAMPAIO et al., 2015). A anilina utilizada é uma solução da marca Synth com pureza 99,8\%.

Para avaliar o efeito inibidor de corrosão da Mangiferina em comparação com a anilina, foram realizados testes de perda de massa e eletroquímicos utilizando solução aquosa de $\mathrm{HCl} 0,5 \mathrm{~mol} . L^{-1}$ na ausência e presença de Mangiferina e anilina, nas concentrações de 0 e $100 \mathrm{ppm}$.

Nos ensaios de perda de massa, foram utilizadas amostras de aço-carbono de dimensões $(25 \mathrm{~mm}$ x 20 $\mathrm{mm} \times 1 \mathrm{~mm})$. As mesmas foram lixadas com lixas d'agua de granulometria de 100 mesh utilizando politriz com rotação aproximada de $300 \mathrm{rpm}$. Posteriormente, as superfícies foram lavadas com água deionizada e etanol e secas com auxílio de ar quente. O procedimento adotado para este ensaio foi o mesmo apresentado na literatura (BARROS et al. 2015), onde é descrito as dimensões e tratamento das superfícies em estudo. As amostras foram imersas em solução de $\mathrm{HCl}$ (0,5 mol. $\left.\mathrm{L}^{-1}\right)$ até um intervalo de tempo de 144 horas na temperatura ambiente na ausência e presença de Mangiferina e anilina nas concentrações de 0 e 100ppm. A perda de massa foi determinada, gravimetricamente, empregando uma balança analítica com precisão de 0,1 $\mathrm{mg}$. Os ensaios foram realizados em triplicatas. A eficiência de inibição $(\mathrm{n} \%)$ foi determinada pela Equação $1:$

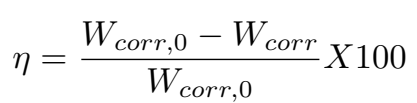

em que, $W_{\text {corr }, 0}$ e $W_{\text {corr }}$ representam a perda de massa na ausência e presença do inibidor, respectivamente.

Para os ensaios eletroquímicos, as amostras de açocarbono foram devidamente cortadas e conectadas a um fio de cobre encapado por intermédio de solda e, posteriormente, embutidas a frio, utilizando resina do tipo epóxi. As superfícies utilizada para o estudo foram lixadas com lixas d'água de granulometria de 100 até 600 mesh, utilizando politriz com rotação aproximada de $300 \mathrm{rpm}$ até o desaparecimento dos traços da lixa anterior. Posteriormente, as superfícies foram lavadas com água deionizadas e etanol. Em seguida foram secos com fluxo de ar quente, conforme apresentado na li- 
teratura (BARROS et al. 2015). As amostras (Eletrodo de Trabalho) tiveram as suas bordas isoladas com esmalte, para evitar infiltração. Após a secagem, as áreas expostas foram devidamente medidas e calculadas.

Os ensaios eletroquímicos foram realizados utilizando um potenciostato/galvanostato AUTOLAB modelo PGSTAT 128N. O software utilizado para o monitoramento foi o NOVA 1.8. Também foi utilizada uma célula padrão de três eletrodos: o eletrodo $\mathrm{Ag} / \mathrm{AgCl} / \mathrm{KCl}$ saturado como eletrodo de referência, um contra-eletrodo de platina, com área exposta superior a três vezes a do eletrodo de trabalho e o eletrodo de trabalho, propriamente dito. Os ensaios foram realizados em célula eletroquímica de capacidade de $250 \mathrm{ml}$, contento a solução $\mathrm{HCl} 0,5$ mol.L ${ }^{-1}$ na ausência e presença de Mangiferina e anilina, nas concentrações de 0 e 100 ppm. Os ensaios foram todos independentes e após cada ensaio a superfície do eletrodo de trabalho era novamente preparada para ser utilizada em outro novo ensaio.

Inicialmente, foi realizado o ensaio de estabilização dos Potenciais de Circuito Aberto (PCAs) para verificar o tempo em que se estabilizava o sistema. Neste ensaio foi monitorado o tempo com relação à variação do PCAs.

Os últimos ensaios realizados, foram os de Polarização Potenciodinâmica onde a varredura iniciou-se a partir de uma sobrevoltagem de $-200 \mathrm{mV}$ a partir do PCA. Antes de iniciar o ensaio, o sistema foi estabilizado por 10 minutos. A taxa de varredura foi de 2,0 $\mathrm{mV} . \mathrm{s}^{-1}$.

Todos os resultados eletroquímicos tiveram seus resultados ajustados de acordo com a área de cada eletrodo de trabalho. Para fins de validação, todos os ensaios eletroquímicos foram realizados em triplicata.

\section{RESULTADOS E DISCUSSÕES}

Os ensaios foram todos realizados seguindo a metodologia já estabelecida nas concentrações de 0 e 100 ppm. Para o teste de perda de massa foi gerado os resultados expostos na figura 1

Observando a figura 1 , podemos notar que houve uma redução significativa da perda de massa relativa com a adição de Mangiferina. A perda de massa para a maior concentração de Mangiferina (100 ppm) reduziu em $68,5 \%$ a ação corrosiva do meio, quando comparada com a amostra sem adição de inibidor. Este resultado sugere que a Mangiferina apresenta comportamento inibidor no estudo proposto.

Quando comparamos a perda de massa da mangiferina com a da anilina que reduziu a perda em 57,09\% em relação a concentração de 0 ppm, vemos que a man-
Figura 1: Ensaio de perda de massa para aço carbono em ácido clorídrico $0,5 \mathrm{~mol} . \mathrm{L}^{-1}$ na ausência e presença de extrato etanólico de Mangiferina e anilina nas concentrações de 0 e 100ppm à temperatura ambiente

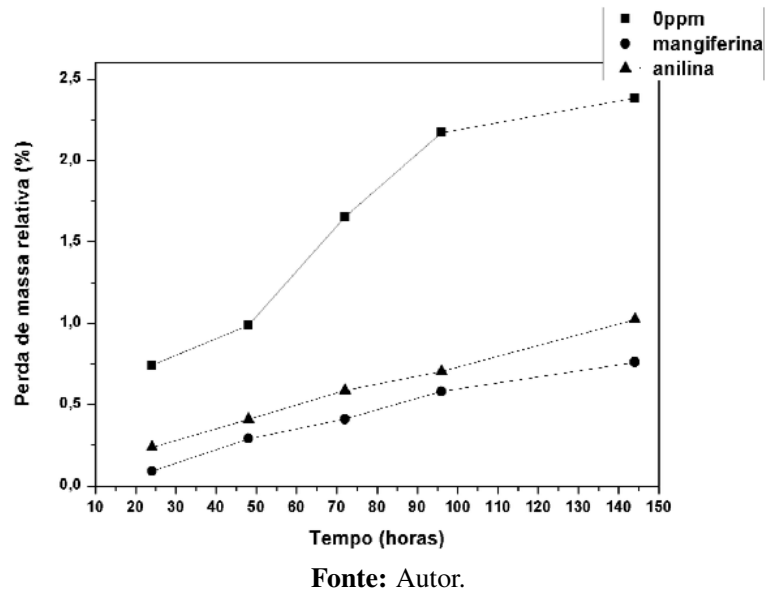

giferina se mostrou mais eficiente neste sistema proposto.

Os resultados apresentados acima estão coerentes com a literatura (BARROS et al. 2015). Segundo os autores Boumhara et al. (2015), o estudo demostrou que o óleo essencial reduziu a perda de massa em $92 \%$ com uma concentração de 3000 ppm de óleo essencial de Artemisia Mesatlantica (AMEO).

Nos ensaios de monitoramento dos PCAs com o tempo, as amostras foram imersas nas soluções ácidas na ausência e na presença de Mangiferina e de anilina em concentrações específicas (0 e 100 ppm), respectivamente. Na figura2, está exposto o monitoramento dos potenciais de circuito aberto (PCA) com um tempo de imersão de 60 minutos.

Visualizando a figura 2, nota-se que os PCAs, após um intervalo de tempo de $10 \mathrm{~min}$ apresentam valores de potenciais praticamente constantes com os exibidos para um ensaio de até 1 hora (60 min.). Este resultado sugere que os sistemas já se encontram estabilizados cineticamente para um tempo de imersão de 10 minutos (MACDONALD, 1987).

Os ensaios de polarização potenciodinâmica foram realizados após 10 minutos de estabilização. Na figura 3, estão apresentados os resultados obtidos para este ensaio nas concentrações de 0 e 100ppm de Mangiferina e de anilina.

Visualizando as curvas de polarização expostas na figura 3, observa-se o deslocamento dos PCAs para valores mais catódicos com aumento da concentração de Mangiferina e de anilina na solução ácida. Também, pode ser observado uma redução das densidades de corrente na região anódica com o aumento da concentração 
Figura 2: monitoramento do potencial de circuito aberto para aço carbono em ácido clorídrico 0,5 mol. $\mathrm{L}^{-1}$ na ausência e presença de extrato etanólico de Mangiferina e de anilina nas concentrações de 0 e 100ppm à temperatura ambiente.

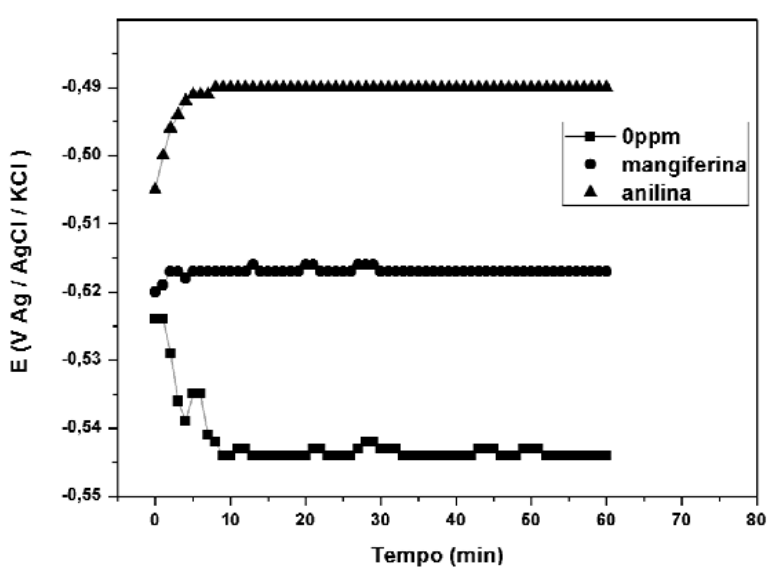

Fonte: Autor.

Figura 3: Curvas de polarização potenciodinâmica obtidas para aço carbono em ácido clorídrico 0,5 mol. $\mathrm{L}^{-1}$ na ausência e presença de Mangiferina e anilina nas concentrações de 0 e 100ppm à temperatura ambiente.

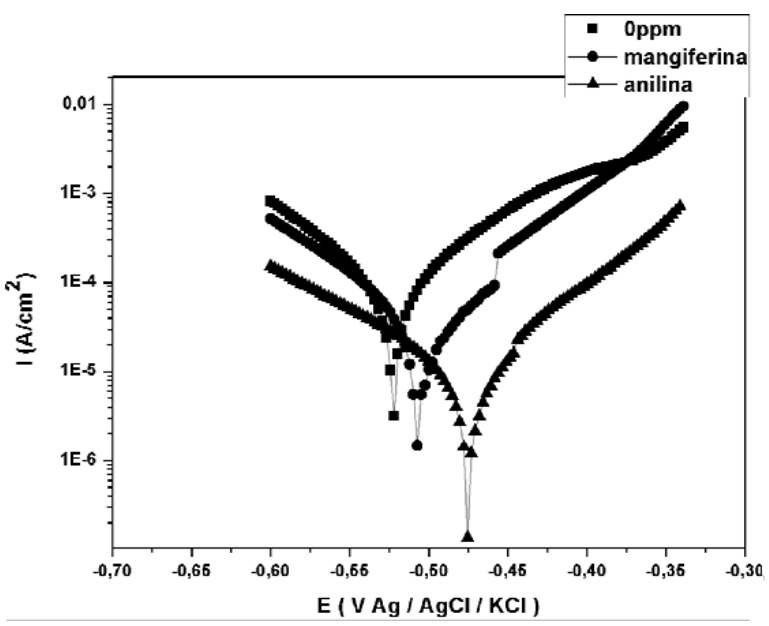

Fonte: Autor.

de mangiferina e de anilina no meio ácido em relação à concentração de $0 \mathrm{ppm}$. Este resultado sugere que o inibidor proposto (Mangiferina) apresenta atividade inibitória no processo corrosivo do aço-carbono no sistema proposto, estando de acordo com os resultados obtidos de perda de massa apresentados. Como pode ser visualizado, a curva de polarização para a anilina apresenta uma redução maior dos potenciais quando comparada a da mangiferina, o que pode ser atribuído a natureza do átomo de nitrogênio no seu grupamento e também ao menor impedimento histérico da molécula em comparação com a mangiferina. Pode ser observado também nas curvas de polarização potenciodinâmica expostas na figura 3 que a ação do inibidor alterou os valores de densidades de corrente, tanto na região catódica como na região anódica, reduzindo-as. Este resultado em comparação com os da anilina confirma que, como a anilina, a mangiferina apresenta uma inibição do tipo mista como retrata a literatura (SHABANI-NOOSHABADI; S. 2015, VRACAR; DRAZIC, 2002).

\section{CONCLUSÕES}

Conclui-se que a Mangiferina apresenta propriedades inibidoras de corrosão dentro do sistema proposto. Sendo esta comparada a anilina em relação a sua ação sobre o substrato metálico, pode-se dizer que é do tipo misto, pois apresenta semelhança aos resultados da anilina. Para os dois inibidores houve uma redução dos potenciais, tanto anódicos como catódicos para valores mais nobres, o que caracteriza esse tipo de processo.

\section{REFERÊNCIAS}

BARROS, I. B.; MOSCOSO, H. Z. L.; CUSTODIO, D. L.; JUNIOR, V. F. V.; BASTOS, I. N. Casca preciosa (aniba canelilla) como inibidor de corrosão do açocarbono. Revista Virtual de Química - RVQ, v. 6, n. 2, 2015. Disponível em: <http://www.uff.br/rvq,2015>

BOUMHARA, K.; TABYAOUI, M.; JAMA, C.; BENTISS, F. Artemisia mesatlantica essential oil as green inhibitor for carbon steel corrosion in $1 \mathrm{~m} \mathrm{HCl}$ solution: Electrochemical and XPS investigations. Journal of Industrial and Engineering Chemistry, v. 29, p. 146 - 155, 2015. ISSN 1226-086X. Disponível em: <http://www.sciencedirect.com/science/article/pii/ S1226086X15001057>.

CANUTO, K. M. Propriedades químicas e farmacológicas de mangiferina: Um composto bioativo de manga (mangifera indica 1.). Embrapa, v. 21, p. 218, 2009.

Conex. Ci. e Tecnol. Fortaleza/CE, v. 10, n. 5, p. 65 - 69, dez. 2016 
EDDY, N. O.; ODOEMELAM, S. A. Inhibition of the corrosion of mild steel in hcl by sparfloxacin. African Journal of Pure and Applied Chemistry, v. 2, p. 132-138, 2008.

EL-ASHRY, E. H.; EL-NEMIR, A.; ESAWY, S. A.; RAGAB, S. Corrosion inhibitors part ii: quantum chemical studies on the corrosion inhibitions of steel in acidic medium by some triazole, oxadiazole and thiadiazole derivatives. Electrochimica Acta, v. 51, p. 3957-3968, 2006.

GENTIL, V. 6. ed. Rio de Janeiro: Livros Técnicos e Científicos, 2013. 360 p.

MACDONALD, J. 1. ed. New York: Wileyinterscience, 1987. 245 p.

SAMPAIO, C. d. G.; FROTA, L. S.; MAGALHÃES, H. S.; DUTRA, L. M.; QUEIROZ, D. C.; ARAÚJO, R. S.; BECKER, H.; SOUZA, J. R. de; RICARDO, N. M.; TREVISAN, M. T. Chitosan/mangiferin particles for cr (vi) reduction and removal. International journal of biological macromolecules, v. 78, p. 273-279, 2015.

SETHURAN, M. G.; RAJA, P. B. Corrosion inhibition of mild steel by datura metel in acidic medium. Pigm. Resin Technol, v. 34, p. 327-331, 2006.

SHABANI-NOOSHABADI, M.; S, G. M. Santolina chamaecyparissus extract as a natural source inhibitor for 304 stainless steel corrosion in 3.5\% nacl. Journal of Industrial and Engineering Chemistry, v. 31, p. 231-237, 2015.

VRACAR, L. M.; DRAZIC, D. M. Adsorption and corrosion inhibitive properties of some organic molecules on iron electrode in sulfuric acid. Corrosion Science, v. 44, p. 1669, 2002. 